\title{
Fabrication of mucocompressive splints for free gingival grafts via medical engineering in patients with reconstructed mandibles
}

\author{
Takuya Kihara a, Tomoko Ikawa a , Yuko Shigeta a ${ }^{*}$, Shuji Shigemoto a , Eriko Ando a, \\ Shinya Hirai a , Naohiko Harada b , Noboru Kawamura b, Kazutoshi Nakaoka c, \\ Yoshiki Hamada c, Takumi Ogawa ${ }^{\text {a }}$ \\ a Department of Fixed Prosthodontics, School of Dental Medicine, Tsurumi University, Kanagawa, Japan \\ ${ }^{b}$ Dental Technician Training Institute, School of Dental Medicine, Tsurumi University, Kanagawa, Japan \\ ' Department of Oral and Maxillofacial Surgery, School of Dental Medicine, Tsurumi University, Kanagawa, Japan
}

\begin{abstract}
Purpose: Acquiring adequate attached mucosa is important in restoring masticatory function with a removable dental prosthesis or dental implant. In patients with inadequate attached mucosa, a free gingival graft (FGG) with a custom stent is used. However, it is challenging to apply the conventional method for fabricating a stent with a reshaped cast in patients with reconstructed mandibles because the reconstructed mandible has limitations due to a titanium mesh, skin flap, and unique bone morphology. In the present report, we have proposed a new design and fabrication process for a mucocompressive splint via medical engineering to acquire the attached mucosa for the prosthodontic treatment of reconstructed mandibles.

Methods: Three-dimensional (3D) craniomandibular models were reconstructed from a computed tomography dataset after mandibular reconstruction. The dentition region was replaced with highly precise scan data of the dental casts using a 3D scanner. The alveolar ridge mucosa and teeth were ideally designed using the 3D model while referring to the anatomical structures. The jig used to fabricate the working dental cast with artificial gum in real space was designed and fabricated using a 3D printer. The alveolar ridge was altered into a simulated configuration on the articulator using a jig. A mucocompressive splint was fabricated on an altered dental cast. The splint was immediately seated on the mandible without any major adjustments after the FGG.

Conclusion: Adequate attached mucosa for the prosthodontic treatment of a reconstructed mandible was obtained by the pre-surgical fabrication of a mucocompressive splint through medical engineering.
\end{abstract}

Keywords: Splint, Attached mucosa, CT, 3D model, Free gingival grafts

\section{Introduction}

Mandibular discontinuity defects can result from benign or malignant tumor resections. Without mandibular reconstruction, oral dysfunction and cosmetic disturbances may occur, leading to a decline in the quality of life[1]. Mandibular reconstruction with a custom-made titanium mesh tray and particulate cancellous bone and marrow (PCBM) restores oral function and the facial esthetic form[2-4]. In our previous studies, a custom-made titanium mesh tray fabricated on a three-dimensional (3D) mandibular plaster model based on the results of virtual simulation prior to surgery was proposed, and its clinical utility was reported[5]. Facial esthetic dissatisfaction improved with surgical treatment. Subsequently, prosth-

DOI: https://doi.org/10.2186/jpr.JPR_D_21_00160

*Corresponding author: Yuko Shigeta, Department of Fixed Prosthodontics, School of Dental Medicine, Tsurumi University, 2-1-3 Tsurumi, Tsurumi-ku, Yokohama, Kanagawa 230-8501, Japan.

E-mail address: shigeta-y@tsurumi-u.ac.jp

Copyright: @ 2022 Japan Prosthodontic Society. All rights reserved. odontic treatment using removable dentures or dental implants contributed to the restoration of masticatory function.

Acquiring adequate attached mucosa and a suitable alveolar ridge form is important to restore masticatory function with a removable dental prosthesis or dental implant. A free gingival graft (FGG) is a mucogingival surgical procedure performed to increase the attached mucosa in patients with localized gingival recession[6,7]. In addition, an FGG can be used to obtain the attached mucosa on the alveolar ridge in a reconstructed mandible. However, the width of the attached mucosa, which is acquired with an FGG, decreases over time[8] and leads to peri-implantitis and poor denture stability. In a previous systematic review, it was reported that the percentage shrinkage of keratinized tissue after an FGG on a partially edentulous area ranged from $2 \%$ to $50 \%$ in the 1- to 12 -month follow-up period[9]. Thus, after an FGG, a mucocompressive splint should be immediately inserted to avoid a remarkable relapse of the attached mucosa. In 2010, Hassani et al.[10] proposed the insertion of a custom stent after an FGG for dental implant treatment in patients with an inadequate attached gingival zone. The stent was fabricated on 


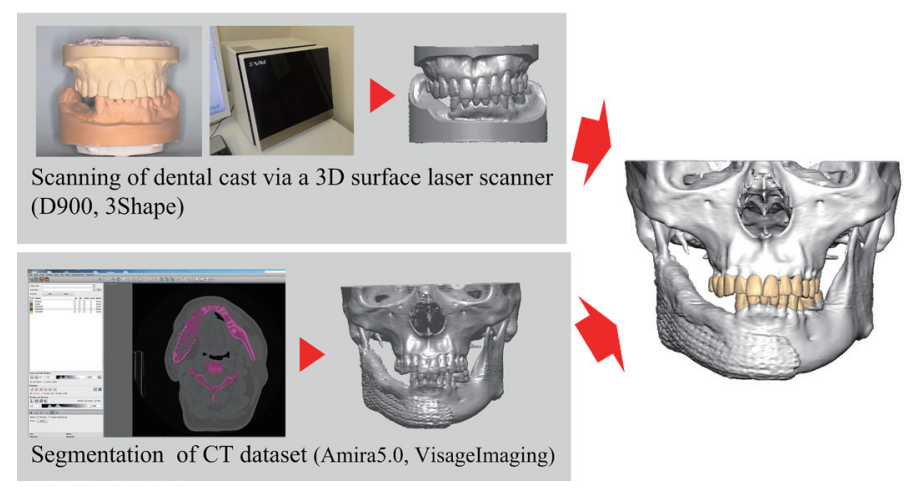

Fig. 1. Data acquisition for fabricating three-dimensional craniomandibular models with a highly precise dentition.

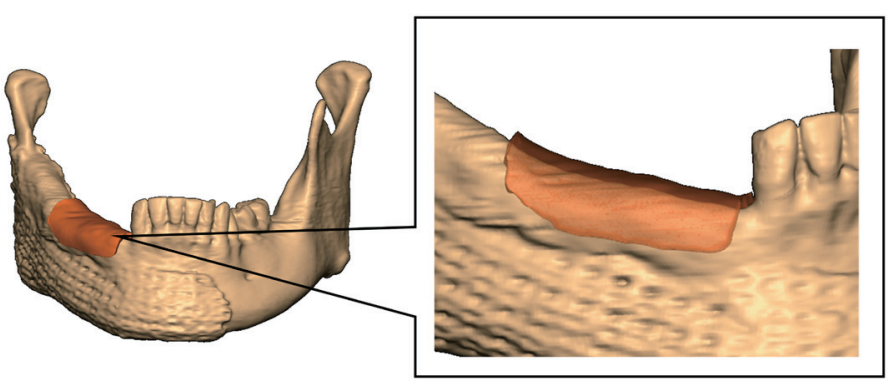

Fig. 2. Designing the alveolar ridge mucosa in the virtual reality space.

the reshaped cast and attached to dental implant abutments after inserting the dental implants. They reported that all grafts were maintained in good condition at the time of stent removal. Their simulation method using a reshaped cast may be sufficient because the patient's mandible was in the dissection form. However, it is challenging to apply their simulation method using a reshaped cast for patients with reconstructed mandibles because the reconstructed mandible has limitations due to a titanium mesh and unique bone morphology and mucosa.

In the present report, we have proposed a new design and fabrication process for a mucocompressive splint via medical engineering to acquire attached mucosa for the prosthodontic treatment of reconstructed mandibles.

\section{Materials and Methods}

2.1. Data acquisition for the fabrication of highly precise craniomandibular 3D models

After reconstructive surgery for mandibular discontinuity defects, computed tomography (CT) was performed using a medical spiral CT scanner (Supria, Hitachi Corp., Tokyo, Japan). The scanning parameters were as follows: tube voltage, $120 \mathrm{kV}$; tube current, 50 $\mathrm{mA}$; scanning speed, $1 \mathrm{~mm} / \mathrm{s}$; scan thickness, $1 \mathrm{~mm}$; pixel size, reconstruction in the range of $0.5 \mathrm{~mm}$. The obtained CT dataset was semi-automatically segmented based on Hounsfield units and reconstructed into 3D models of the cranium and mandible with a titanium mesh tray using image analysis software (Amira 5.0, Thermo Fisher Scientific, Waltham, MA) in the virtual reality (VR) space. The cranial and mandibular models involved the respective dentition.

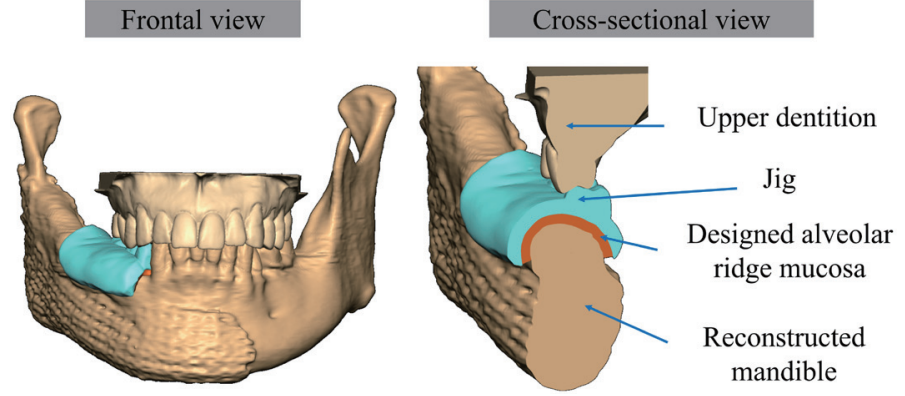

Fig. 3. Frontal and cross-sectional view of the alveolar ridge mucosa and jig simulated in the virtual reality space.
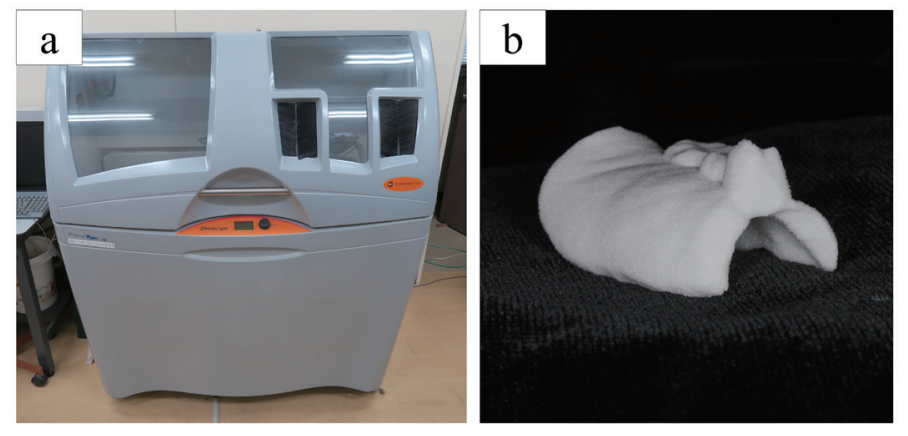

Fig. 4. a) Three-dimensional printer (Z Printer 450, 3D Systems), b) Jig.

However, the dentition was insufficiently reproduced because of the spatial resolution of the $\mathrm{CT}$ and metal artifacts.

To improve the reproducibility of the dentition, the upper and lower casts were scanned with a 3D surface laser scanner (D900, 3Shape, Copenhagen, Denmark), and the 3D-reconstructed cast models were merged into the bone models. The dentition reconstructed using the CT dataset was used for registration with the scanned cast data using the iterative closest point algorithm and was deleted and replaced with the precise cast models using a 3D processing software (Rapidform 2006, INUS Technology, Seoul, Korea) (Fig. 1)[11].

\subsection{Designing the alveolar ridge mucosa and jig for the altered cast}

The area of the alveolar ridge mucosa was ideally designed using the 3D mandibular model based on the definition of the denture base outline. The thickness of the alveolar ridge mucosa was adjusted to $1.5-3.0 \mathrm{~mm}$ within the physiological range[12], while referring to the volume, height, and surface irregularities of the alveolar ridge in the reconstructed mandibles (Fig. 2). The mucobuccal fold was designed as deep as possible above the superior margin of the titanium mesh tray, considering the amount of shrinkage after an FGG. The defective teeth were restored by mirroring the teeth on the unaffected side in the VR space. Occlusal contacts were designed according to the removable denture to compress the grafted mucosa suitably. Using the 3D model, a jig that was used to fabricate the working dental cast with artificial gum in real space was designed (Fig. 3). These simulations were performed using 3D processing software (FreeForm, 3D Systems, Rock Hill, SC, USA). The jig was fabricated using a 3D printer (Z Printer450, 3D Systems, Rock Hill, SC) with plaster materials (Fig. 4). The layer thickness was set at $0.1 \mathrm{~mm}$. 

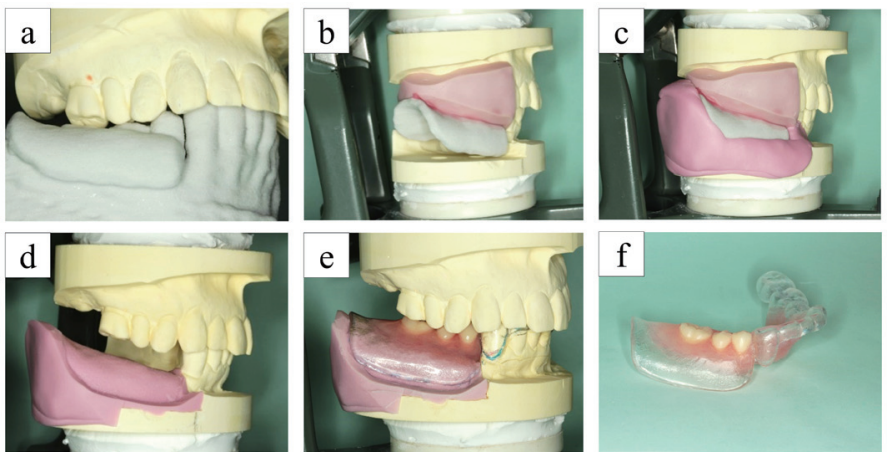

Fig. 5. Fabrication workflow of the mucocompressive splint.

a) Recording of the positional relationship between the upper dentition and the jig on the simulated mandibular model, b) Attachment of the jig to the upper dental cast on the articulator using the putty (pink), c) Filling silicone, d) Alteration of the alveolar ridge in the lower dental cast using the jig and silicone putty (pink), e) Fabrication of the mucocompressive splint with a polyester sheet, self-curing resin, and artificial teeth on the altered dental cast, f) The mucocompressive splint.

\subsection{Fabrication of the mucocompressive splint}

The fabrication workflow of the mucocompressive splint is shown in Figure 5. First, the positional relationship between the upper cast and the jig on the simulated mandibular model, which was fabricated using a 3D printer, was recorded with silicone putty (Zetalabor, Zhermack, Italy). The upper and lower casts were mounted on the articulator, and the alveolar ridge from the lower cast targeted for the FGG was removed. The jig was attached through the silicone record, and the silicone material was inserted between the jig and the lower cast to represent the simulated alveolar mucosa in the working cast. The mucocompressive splint was fabricated with a polyester sheet (Duran $2.0 \mathrm{~mm}$, Scheu, Germany), self-curing resin (ParaXpress, Heraeus-Kulzer Co., Hanau, Germany), and artificial teeth (Veracia SA A3, Shofu, Japan) on the altered dental cast.

The splint was immediately seated on the mandible without any major adjustments after the FGG. After inserting the splint, the occlusion and flange of the denture base were slightly adjusted a few times during the healing process of the grafted mucosa. The occlusal surface of the remaining teeth was covered with a splint with a 2-mm thick polyester sheet, and occlusal adjustments were performed on the working casts before wearing them, in advance. After intra-oral occlusal adjustment, the purpose, precautions, and period of use were explained to the patients. They were specifically instructed that the intake of excessive hard food on the FGG side should be avoided. The mucocompressive splint was used for approximately two months post-FGG. The patient removed it after eating and had it cleaned. Except in this situation, the patients continued to wear the splints regularly during this period.

Our mucocompressive splint was designed in the same manner as the occlusal overlay removable partial denture. Thus, this splint demonstrated a compressive effect on the grafted mucosa by biting and splinting the remaining teeth. Naturally, the health of the remaining teeth was maintained due to the perioperative care preand post-operation, preventing the mucocompressive splint from deviation, even if the patient had few remaining teeth.

\section{Difference from Conventional Methods}

In mandibular reconstruction using the tibia or titanium mesh for patients with malignant tumors, such as squamous cell carcinoma (SCC), a skin flap is grafted onto the reconstructed alveolar ridge[13]. An FGG after mandibular reconstruction can help acquire the attached mucosa, which is suitable for prosthodontic treatments after mandibular reconstruction. In 2021, Li et al.[14] reported that an FGG is recommended to avoid peri-implantitis and bone loss in patients with a reconstructed jaw. They carried out an FGG after inserting dental implants into the grafted bone.

The removable partial denture should be promptly placed to restore masticatory function after mandibular reconstruction prior to dental implant treatment, and the dental implant insertion should be performed by referring to its occlusal relationship based on a topdown approach. In addition, some patients did not expect dental implant treatment. Therefore, it is important to acquire the attached mucosa to ensure denture stability. Therefore, we performed an FGG before the dental implant insertion. In a previous study, the stent had a non-compressive area around the abutment to secure sufficient blood circulation during the healing period after an FGG[14]. In our method, the risk of graft shrinkage may be increased compared to that in their methods because the repair mechanism after an FGG is different in the presence or absence of an abutment. On the other hand, our method, which was carried out prior to dental implant insertion, may have an advantage over their method of compressing the grafted mucosa with uniform pressure due to occlusal force with regard to graft shrinkage. Adequate mucosa was obtained using the proposed method.

In a previous study, a reshaped cast was used to fabricate stents[10]. In contrast, medical engineering techniques were applied to our simulation. The cast has an advantage over the 3D model reconstructed from the CT dataset with regard to occlusal reconstruction because the 3D CT model is affected by image resolution and metal artifacts. Therefore, our 3D model was produced to merge the reconstructed bone model from the $\mathrm{CT}$ dataset and scanned cast data. In addition, the VR simulation was applied to design an ideal alveolar ridge mucosa shape because it is challenging to estimate the reconstructed bone shape, which deviates from the dissection form, from the shape of the skin flap surface.

As a result, our mucocompressive splint could provide a suitable occlusal relationship, not only an ideal impression surface.

\section{Effect or Performance}

Six patients underwent mandibular segmental resections and reconstructions due to SCC (1 case), fibrous dysplasia (1 case), and ameloblastoma (4 cases). An FGG with a mucocompressive splint, designed and fabricated via the medical engineering approach mentioned above, was used in this case. Figure $\mathbf{6}$ and $\mathbf{7}$ show two clinical cases: Case 1: SCC and Case 2: fibrous dysplasia. After surgical mandibular reconstruction using the custom-made titanium mesh tray and PCBM, an FGG was performed, and a mucocompressive splint was inserted. Cases 1 and 2 used a mucocompressive splint for 56 and 82 days, respectively. Using a mucocompressive splint, the healing of the grafted mucosa was induced to the ideal configuration for prosthodontic treatment. 


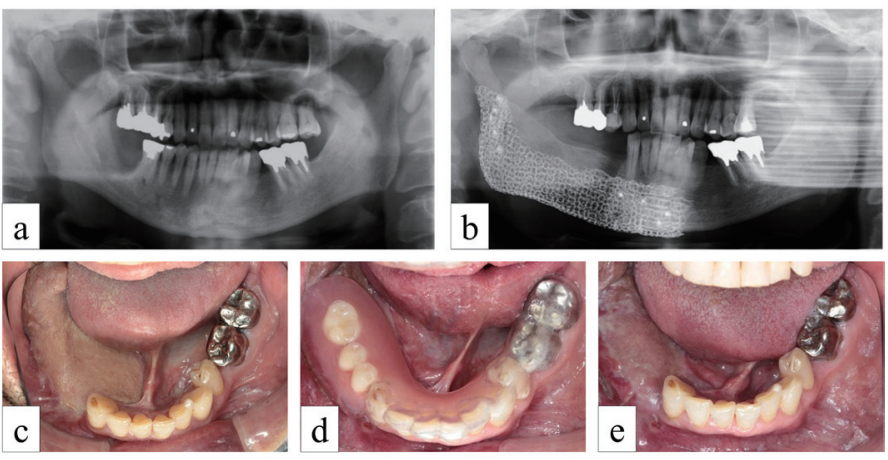

Fig. 6. Clinical case I: Patient with gingival carcinoma (malignant tumor) who underwent a free gingival graft (FGG) surgery along with a mucocompressive splint.

a) Panoramic radiograph before the mandibular reconstruction, b) Panoramic radiograph after the mandibular reconstruction, c) Intraoral photograph before the FGG, d) Intraoral photograph with the mucocompressive splint, e) Intraoral photograph after the FGG.
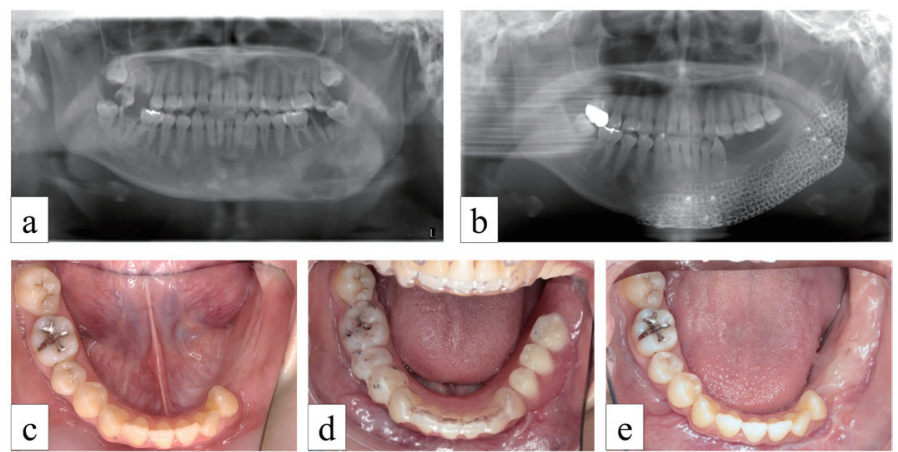

Fig. 7. Clinical case II: Patient with fibrous dysplasia (benign tumor) who underwent a free gingival graft (FGG) surgery along with a mucocompressive splint.

a) Panoramic radiograph before the mandibular reconstruction, b) Panoramic radiograph after the mandibular reconstruction, c) Intraoral photograph before the FGG, d) Intraoral photograph with the mucocompressive splint, e) Intraoral photograph after the FGG.

contribution of the splint was evaluated by observing the mucogingival junction using a histochemical staining method with iodide solution[15,16] (Fig. 8). Excessive relapsing of the attached mucosa could be avoided because the grafted mucosa was adequately compressed, and the attached mucosa was suitably maintained after wearing the prosthesis. The buccolingual width of the attached mucosa, acquired through the FGG, was measured on the intraoral images using an image analysis software program (Photoshop CS2, Adobe, CA, US). The results are shown in Table 1. The actual width of the attached mucosa might be larger than the measured value because of the two-dimensional image analysis. The width of the attached mucosa was larger than the average crown width[17] (Table 1). Thus, the attached mucosa was suggested to be sufficiently acquired for application to dental implants or removable partial dentures.

The patients had no pain or discomfort while wearing a mucocompressive splint. They did not complain of any inconvenience regarding mastication with the splint. In addition, the splint could provide a stable occlusal relationship during the healing period of

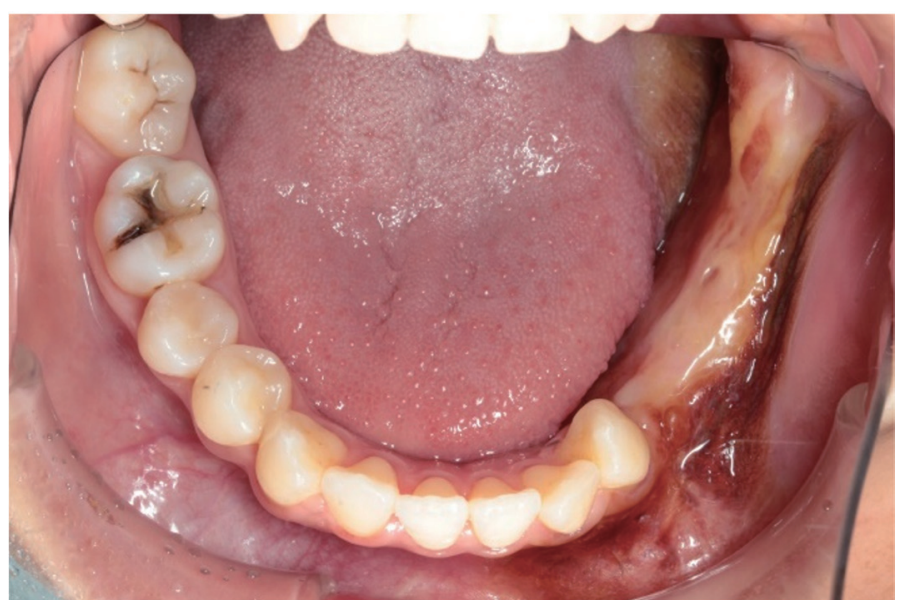

Fig. 8. Evaluation of the mucogingival junction using a histochemical staining method with iodide solution.

Table 1 Width of the attached mucosa measured using two-dimensional image analysis and reference of the crown width

\begin{tabular}{lccc}
\hline & $\begin{array}{c}\text { Anterior } \\
\text { region }\end{array}$ & $\begin{array}{c}\text { Premolar } \\
\text { region }\end{array}$ & $\begin{array}{c}\text { Molar } \\
\text { region }\end{array}$ \\
\hline $\begin{array}{l}\text { Width of the attached } \\
\text { mucosa }(\mathrm{mm})\end{array}$ & $9.38 \pm 2.41$ & $9.33 \pm 2.08$ & $10.9 \pm 2.76$ \\
Crown width $(\mathrm{mm})$ & $6.5^{17)}$ & $8.0^{17)}$ & $10.8^{17)}$ \\
\hline
\end{tabular}

17) Fujita T. Textbook of dental anatomy. 22nd ed. Tokyo: Kanehara; 1995.

the FGG and could be utilized to design the final prosthesis. The duplicated splint can be used as a stent to insert dental implants.

\section{Conclusion}

A suitable alveolar ridge mucosa for prosthodontic treatment was obtained by the pre-surgical fabrication of a mucocompressive splint through medical engineering. The attached mucosa was suitably maintained in patients with reconstructed mandibles after wearing the final prosthesis.

\section{Conflicts of interest}

The authors declare no conflicts of interest.

\section{References}

[1] Goh BT, Lee S, Tideman H, Stoelinga PJW. Mandibular reconstruction in adults: a review. Int J Oral Maxillofac Surg. 2008;37:597-605. https://doi. org/10.1016/j.jjom.2008.03.002, PMID:18450424

[2] Lee W, Choi W, Lee H, Choi N, Hwang D, Kim U. Mandibular reconstruction with a ready-made type and a custom-made type titanium mesh after mandibular resection in patients with oral cancer. Maxillofac Plast Reconstr Surg. 2018;40:35. https://doi.org/10.1186/s40902-018-0175-z, PMID:30538971

[3] Ozaki H, Sakurai H, Yusa K, Kitabatake K, Kobayashi T, lino M. Mandibular reconstruction with fibula bone graft followed by particulate cancellous bone and marrow graft with titanium mesh tray. J Oral Implantol. 2016;42:381-4. https://doi.org/10.1563/aaid-joi-D-16-00009, PMID:27077954 
[4] Ikawa T, Shigeta Y, Hirabayashi R, Hirai S, Hirai K, Harada N, et al. Computer assisted mandibular reconstruction using a custom-made titan mesh tray and removable denture based on the top-down treatment technique. J Prosthodont Res.2016;60:321-31.https://doi.org/10.1016/j.jpor.2016.01.009, PMID:26895971

[5] Yamada H, Nakaoka K, Sonoyama T, Kumagai K, Ikawa T, Shigeta Y, et al. Clinical usefulness of mandibular reconstruction using custom-made titanium mesh tray and autogenous particulate cancellous bone and marrow harvested from tibia and/or ilia. J Craniofac Surg. 2016;27:586-92. https:// doi.org/10.1097/SCS.0000000000002472, PMID:27092909

[6] Shah R, Thomas R, Mehta D. Recent modifications of free gingival graft: A case series. Contemp Clin Dent. 2015;6:425-7. https://doi.org/10.4103/0976237X.161910, PMID:26321849

[7] Marin DOM, Leite ARP, Nícoli LG, Marcantonio C, Compagnoni MA, Marcantonio $E$ Jr. Free gingival graft to increase keratinized mucosa after placing of mandibular fixed implant-supported prosthesis. Case Rep Dent. 2017;2017:1-5. https://doi.org/10.1155/2017/5796768, PMID:28293441

[8] Karakış Akcan S, Güler B, Hatipoğlu H. The effect of different gingival phenotypes on dimensional stability of free gingival graft: A comparative 6-month clinical study. J Periodontol. 2019;90:709-17. https://doi. org/10.1002/JPER.18-0530, PMID:30648265

[9] Thoma DS, Buranawat B, Hämmerle CHF, Held U, Jung RE. Efficacy of soft tissue augmentation around dental implants and in partially edentulous areas: a systematic review. J Clin Periodontol. 2014;41(suppl 15):S77-91. https://doi.org/10.1111/jcpe.12220, PMID:24641003
[10] Hassani A, Sadrimanesh R, Vahdati SA, Sadr-eshkevari P. Free gingival graft immobilization: a pilot study on a newly designed stent. J Oral Implantol. 2010;36:123-30. https://doi.org/10.1563/AAID-JOI-D-09-00034, PMID:20426589

[11] Kihara T, Tanimoto K, Michida M, Yoshimi Y, Nagasaki T, Murayama T, et al. Construction of orthodontic setup models on a computer. Am J Orthod Dentofacial Orthop. 2012;141:806-13. https://doi.org/10.1016/j. ajodo.2011.10.027, PMID:22640682

[12] Takahashi A, Morita K, Hirakawa K, Toyoda M. Measuring the thickness of the lower jaw membrane by Schleimhaut-Dicken-Meß. Nippon Hotetsu Shika Gakkai Zasshi. 2002;46:341-6. https://doi.org/10.2186/jjps.46.341

[13] Kumar BP, Venkatesh V, Kumar KAJ, Yadav BY, Mohan SR. Mandibular Reconstruction: overview. J Maxillofac Oral Surg. 2016;15:425-41. https://doi. org/10.1007/s12663-015-0766-5, PMID:27833334

[14] Li R, Meng Z, Zhang Y, Shan X, Wang Y, He Y. Soft tissue management: a critical part of implant rehabilitation after vascularized free-flap reconstruction. J Oral Maxillofac Surg. 2021;79:560-74. https://doi.org/10.1016/j. joms.2020.11.006, PMID:33279473

[15] Hsu YT, Wang HL. Clinical assessment of the gingiva and alveolus. In: Karateew ED, editor. Implant Aesthetics: Keys to Diagnosis and Treatment, Switzerland. Springer International Publishing AG; 2017; 109-10.

[16] Bhatia G, Kumar A, Khatri M, Bansal M, Saxena S. Assessment of the width of attached gingiva using different methods in various age groups: $A$ clinical study. J Indian Soc Periodontol. 2015;19:199-202. https://doi. org/10.4103/0972-124X.152106, PMID:26015672

[17] Fujita T. Textbook of dental anatomy. 22nd ed. Tokyo: Kanehara; 1995.

This is an open-access article distributed under the terms of Creative Commons Attribution-NonCommercial License 4.0 (CC BYNC 4.0), which allows users to distribute and copy the material in any format as long as credit is given to the Japan Prosthodontic Society. It should be noted however, that the material cannot be used for commercial purposes. 